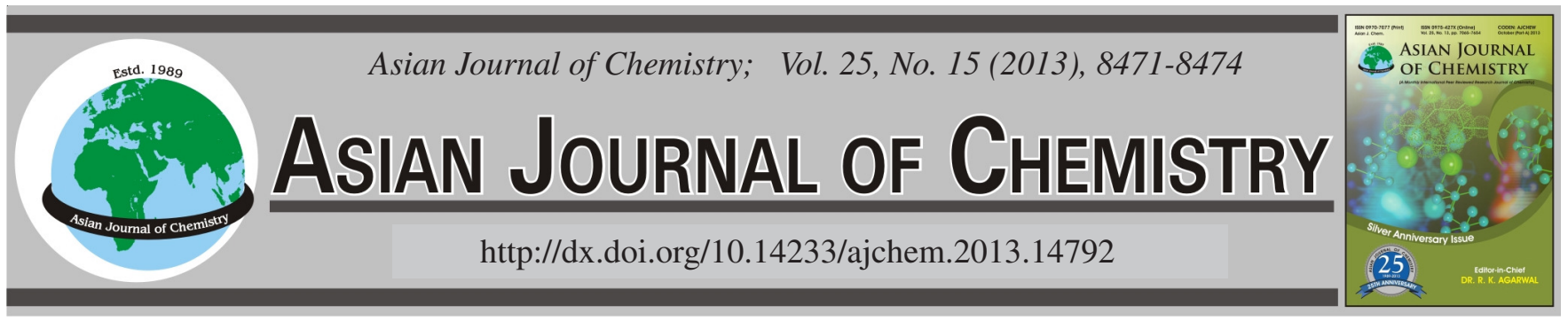

\title{
Kinetic of Nitrate Reduction by Nanoscale Zero-Valent Iron on the Basis of Different Models Estimation
}

\author{
JiAnShe TANG*, Li XIANG and Feng ZhaO
}

School of Environment and Energy Engineering, Anhui University of Architecture, 292 Ziyun Road, Economic-Technological Development Area, Hefei 230601, P.R. China

*Corresponding author: Fax: +86 551 3828123; Tel: +86 551 3828122; E-mail: tjs28@aiai.edu.cn

(Received: 7 November 2012;

Accepted: 24 August 2013)

AJC-13976

\begin{abstract}
In this study, a more fine-grained spherical nanoscale zero valent iron (nZVI) with diameter of 20-50 nm was synthesized in ethanol-water mixed solvent. The structures and physical properties of nZVI were characterized by transmission electron microscope, X-ray diffractometer analyzer. Reactivity of the nZVI was affirmed by denitrification of nitrate. The removal efficiency of nitrate in the presence of nZVI was $80 \%$ within $1 \mathrm{~h}$ at optimal conditions. In view of this, the $\mathrm{nZVI}$ is efficient to removing nitrate and offers a great potential to the removal of other contaminants in water. The kinetic estimations in terms of three kinds model (the first-order, the second-order and the LangmuirHinshelwood) under the conditions of different initial nitrate concentration or different nZVI dosage were compared with each other. The result was obtained that the S-model fits the denitrification very well.
\end{abstract}

Key Words: Nanoscale zero valent iron, Nitrate, Kinetic, Reduction.

\section{INTRODUCTION}

Zero-valent iron has received much recent attention due to their low cost, non-toxicity nature, high activity and abundant in nature ${ }^{1}$. Nanoscale zero-valent iron (nZVI) with particle size at the nano-scale exhibits superior activity because of their larger surface area and great intrinsic reactivity of surface sites ${ }^{2}$. Nanoscale zero valent iron technology has been widely investigated for the reductive transformation of a wide range of chemical pollutants, including: antibiotics ${ }^{3}$, azo dyes ${ }^{4}$, pesticides ${ }^{5}$, nitroamines $^{6}$, nitroaromatics ${ }^{7}, 2,4$-dichlorophenol ${ }^{8}$, polychlorinated biphenyls ${ }^{9}$, inorganic anions, including nitrate ${ }^{10}$, perchlorate $^{11}$ and metals, including chromium ${ }^{12}$, copper $^{13}$, cadmium $^{14}$ and $\operatorname{arsenic}^{15}$.

Nitrate pollution of groundwater and surface water has become a serious environment problem in many parts of the world $^{16}$. The removal of nitrate from contaminated wastewater frequently involves microbial denitrification, ion exchange, reverse osmosis, electrocatalytic and high temperature and chemical reduction process ${ }^{17}$.

Chemical reduction process on the basis of nZVI might be relatively efficient for denitrification process. Several research papers reported the reaction mechanisms and pathways in the case of the adaptation of $n Z V^{18-22}$. The reaction mechanism of nitrate removals depending on $\mathrm{pH}$, temperature, both in buffered and unbuffered solutions were revealed in the former reports $^{18,23,24}$.
A wide variety of kinetic models, including first order kinetic model ${ }^{19,25}$, higher reaction order kinetic model ${ }^{26}$, for nitrate reduction by nZVI have been published. The usage of others models including Langmuir ${ }^{27}$ and Michaelis-Menten ${ }^{28}$ were investigated subsequently.

In the present study, we prepared nZVI particles in ethanolwater solution. The present investigation aimed at gaining mechanistic insight into the chemical reduction of nitrate in aqueous solution by synthesized nZVI particles. Specifically, we followed the kinetics of nitrate removal on the basis of the first-order, the second-order kinetic and the LangmuirHinshelwood kinetic models.

\section{EXPERIMENTAL}

All chemical reagents such as potassium borohydride $\left(\mathrm{K}_{\mathrm{B}} \mathrm{H}_{4}\right)$, potassium nitrate $\left(\mathrm{KNO}_{3}\right)$, ferrous sulfate heptahydrate $\left(\mathrm{FeSO}_{4} \cdot 7 \mathrm{H}_{2} \mathrm{O}\right)$, sodium hydroxide $(\mathrm{NaOH})$ and sulfuric acid $\left(\mathrm{H}_{2} \mathrm{SO}_{4}\right)$ and anhydrous ethanol were purchased from Sonipharm Chemical Reagent Co., Ltd (Shanghai, China). All the chemicals obtained above were analytical grade and were used as received without further purification.

Preparation of the nanoscale zero valent iron (nZVI): Nanoscale zero valent iron was synthesized in terms of the procedure outlined in previous literature ${ }^{16}$. In brief, $9 \mathrm{~g}$ of $\mathrm{FeSO}_{4} \cdot 7 \mathrm{H}_{2} \mathrm{O}$ was first dissolved in $400 \mathrm{~mL}$ of deionized water and $100 \mathrm{~mL}$ of alcohol was then added. $2.44 \mathrm{~g}$ of $\mathrm{NaBH}_{4}$ was 
dissolved in $50 \mathrm{~mL}$ of deionized water and dropwise added to the $\mathrm{FeSO}_{4} \cdot 7 \mathrm{H}_{2} \mathrm{O}$ solution that was mixed by an impeller at $500 \mathrm{rpm}$.

The black precipitant was collected using a magnet and washed three times with degassed deionized water and then washed with anhydrous ethanol for at least three times. The whole process was carried out under the protection of nitrogen atmosphere. X-Ray powder diffraction (XRD) was performed on a Bruker D8 diffractometer.

Batch experimental procedures: For the batch test, 200 $\mathrm{mL}$ of reactant solution containing nitrate was added into 500 $\mathrm{mL}$ flasks containing nZVI. The flask was bathed in a water bath for keeping the reaction temperature at $25^{\circ} \mathrm{C}$. The final concentrations of nitrate were 50,100 and $150 \mathrm{mg} / \mathrm{L}$. The final concentrations of nZVI were set as $0.5,2.0$ and $5.0 \mathrm{~g} / \mathrm{L}$.

The dispersion was continuously stirred with a magnetic stirrer and samples were taken periodically at 2, 5, 10, 20, 40, 60, 90 and $120 \mathrm{~min}$. The samples were filtered with $0.45 \mu \mathrm{m}$ syringe filters and analyzed immediately.

To analyze the nitrate and nitrite concentrations, an ion chromatograph equipped with an AS-16 column was adopted. The ammonium was determined by NESSLER's reagent colorimetric method using a UV-9200N spectrophotometer (Lengguang, China).

Kinetic models for reaction: The first-order and secondorder kinetic models were used to fit the experimental data. The two models are described as follow eqns. 1 and 2, respectively.

$$
\begin{aligned}
& r=-\frac{\mathrm{dC}}{\mathrm{dt}}=\mathrm{kC} \\
& \mathrm{r}=-\frac{\mathrm{dC}}{\mathrm{dt}}=\mathrm{kC}^{2}
\end{aligned}
$$

where $r$ is the rate of dye degradation, $\min ^{-1} \mathrm{mg} / \mathrm{L}$; $\mathrm{C}$ is the concentration at any time, $\mathrm{mg} / \mathrm{L}$; $\mathrm{k}$ is the rate constant, $\mathrm{min}^{-1}$. The reactions were also expressed by a more complex kinetic model, namely the Langmuir-Hinshelwood kinetic, which captures the catalytic behavior of many metal catalysts ${ }^{29}$. It takes into consideration the adsorption of both reactants over the Fe surface and reaction between catalyst and nitrate. The reaction rate is described as:

$$
\mathrm{r}=-\frac{\mathrm{dC}}{\mathrm{dt}}=\frac{\mathrm{kKC}}{1+\mathrm{KC}}
$$

where $\mathrm{k}$ and $\mathrm{K}$ are the limiting rate constants of reaction at maximum coverage under the given experimental conditions and equilibrium constant for adsorption of nitrate onto nZVI. Integrating eqns. 1-3 with respect to the limits $C=\mathrm{C}_{0}$ at time $\mathrm{t}=0$ and $\mathrm{C}=\mathrm{C}_{\mathrm{t}}$ at any time $\mathrm{t}$, the formations of all expression can be obtained as follow:

$$
\begin{gathered}
\mathrm{C}_{\mathrm{t}}=\mathrm{C}_{0} \times \exp (-\mathrm{kt}) \\
\mathrm{C}_{\mathrm{t}}=\frac{1}{\mathrm{kt}+\frac{1}{\mathrm{C}_{0}}} \\
\ln \left(\frac{\mathrm{C}_{0}}{\mathrm{C}_{\mathrm{t}}}\right)+\mathrm{K}\left(\mathrm{C}_{0}-\mathrm{C}_{\mathrm{t}}\right)=\mathrm{kKt}
\end{gathered}
$$

The formations can be obtained and were edited into soft of origin 7.5. Then the nitrate reducing reaction data could be fitted automatically by computer on the basis of the 3 models.

\section{RESULTS AND DISCUSSION}

Characterization of nanoscale zero valent iron (nZVI): The XRD pattern of nZVI is presented in Fig. 1. An obvious diffraction peaks at $44.8^{\circ}$ and at $65.2^{\circ}$ can be observed, which are in accordance with the (110) and (200) diffraction of bodycentered cubic $\alpha-\mathrm{Fe}$. The peak pattern of XRD spectra was most close to that of elementary iron rather than other iron oxides and hydroxides.

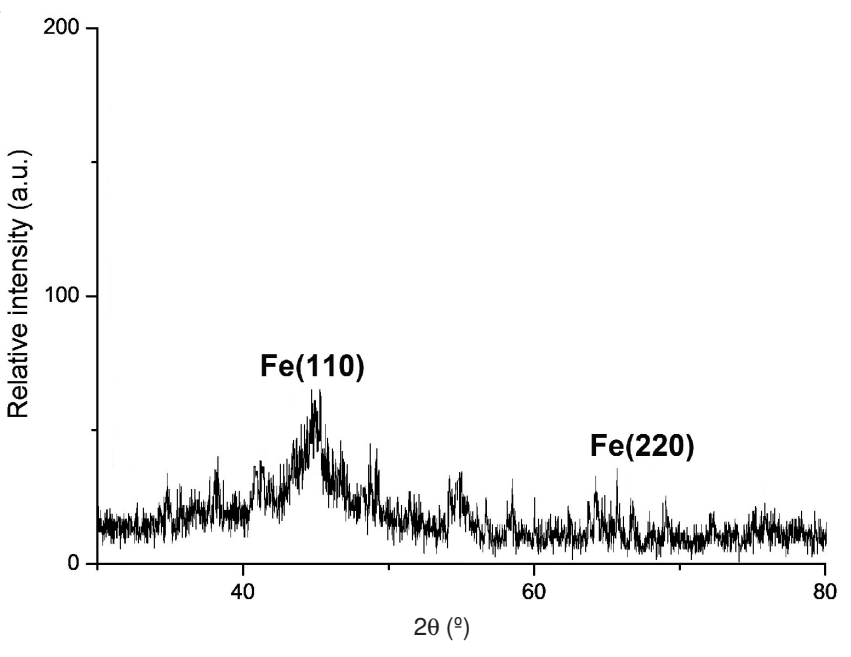

Fig. 1. XRD pattern of fresh nanoscale zero valent iron

Model estimation for nitration reduction: Kinetics studies has significant role for the investigation of the profile of nitration degradation in the presence of catalyst nZVI. The experimental results are presented in Fig. 2. The removal rates of nitrate after $1 \mathrm{~h}$ can reach more than 76.0, 64.6 and $44.7 \%$ with the initial nitrate concentration of 50,100 and $150 \mathrm{mg} / \mathrm{L}$, respectively and then reached 88, 72.7 and $60 \%$ after another $1 \mathrm{~h}$. Nitrate was declined fast in the beginning up to $2 \mathrm{~h}$, but no more than reduction has taken place after that, similar to the result of Kuen-Song $\operatorname{Lin}^{17}$. The nitrate removal rates, which published by Zhang et al. ${ }^{19}$, can reach $96.0 \%$ after $1 \mathrm{~h}$ using nZVI. Hwang et al. ${ }^{21}$ refer firstly that nitrate reduction rate was very fast during the initial $15 \mathrm{~min}$. Therefore, the reaction constant $\mathrm{k}$ was differentiated at two reaction periods of $0-15$ min and $>15 \mathrm{~min}^{21}$. As for the result of our experiments, it was concluded that the nitrate reduction rate was fast at initial periods as well as at low initial nitrate concentration from Fig. 3. These different results could be explained by the different nZVI and different reaction conditions at different initial nitrate concentration.

In order to investigate the mechanism of degradation, the first-order (F-model), the second-order (S-model) and the Langmuir-Hinshelwood (L-H model) kinetics models were used to test the degradation of nitrate at various initial concentration of 50,100 and $150 \mathrm{mg} / \mathrm{L}$ during the reaction under certain conditions (temperature $25^{\circ} \mathrm{C}$, initial $\mathrm{pH}$ value 7.0 , dosage of nZVI $2 \mathrm{~g} / \mathrm{L}$ ). The model estimations for nitration reduction in terms of models are shown in Fig. 2. The dash, 


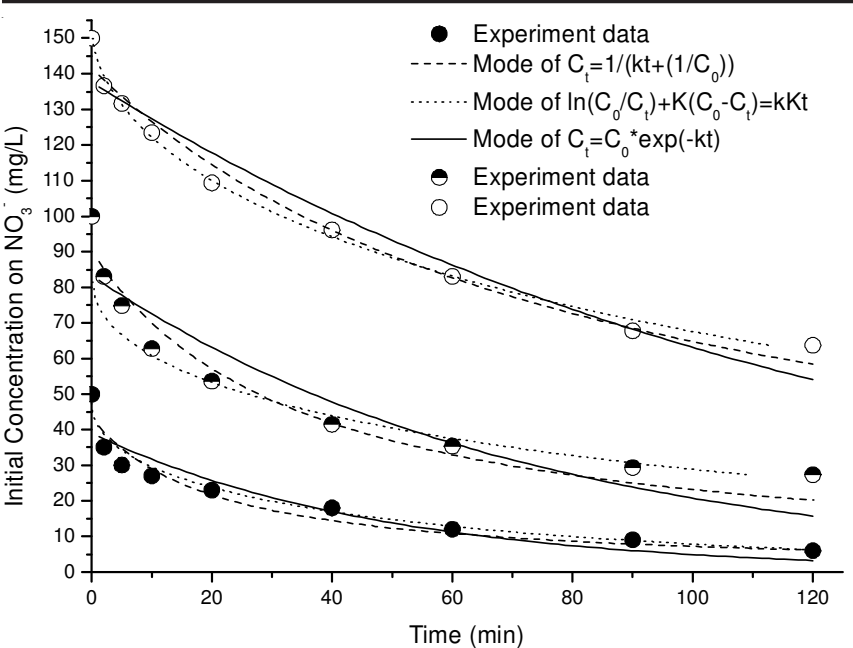

Fig. 2. Kinetic model estimation for nitration reduction at the initial nitration concentration of $50 \mathrm{mg} / \mathrm{L}$ (solid circle), $100 \mathrm{mg} / \mathrm{L}$ (semisolid circle) and $150 \mathrm{mg} / \mathrm{L}$ (hollow circle). The dash lines stand for the model estimation of second-order kinetic. The dot lines stand for the model estimation of L-H kinetic. And the solid lines stand for the model estimation of first-order kinetic

dot and solid lines stand for the S-, L-H and F-kinetics models estimates, respectively. The $\mathrm{R}^{2}$ values of $\mathrm{S}$-model estimates were $0.9771,0.9365$ and 0.9649 at initial nitrate concentration of 50,100 and $150 \mathrm{mg} / \mathrm{L}$, respectively, which were higher than those of F-model and L-H model estimates. Therefore, it was clear that the S-model could be suit for fitting the denitrification within the provided initial nitrate concentration range.

The observed F-, S- and L-H reaction rate constants $\mathrm{k}$ at different initial nitrate concentration are shown in Fig. 3. The constants $\mathrm{k}$ of $\mathrm{F}$ model were decreased from 0.0208, 0.0139 to 0.0078 and those of L-H model were decreased from 0.0210 , 0.0123 to 0.0064 , when the initial nitrate concentration increased from 50,100 to $150 \mathrm{mg} / \mathrm{L}$, respectively. Nevertheless, the $\mathrm{k}$ values of S-model apparent no difference between 3 initial concentrations (Fig. 3). Several studies have been approved that the $\mathrm{k}$ values of $\mathrm{F}$ model were closely at different initial nitrate concentration. The conclusion of denitrification complying with the first-order reaction with respect to nitrate concentration was also point out in these reports ${ }^{16-19,23-25}$. However, Yang and Lee ${ }^{18}$ point out that the first- or pseudo-first-order kinetic model might not be suitable to describe chemical reduction of nitrate by $n Z V I$. In our research, the $\mathrm{k}$ values of $\mathrm{S}$ model were independent of the initial nitrate concentration and the $S$ model was more appropriate to fitting denitrification and is recommended.

Effect of nanoscale zero valent iron Dosage on nitrate reduction: $\mathrm{nZVI}$ dosage, as a significant role for denitrification, would be vital to the removal of nitrate. The denitrification of nitrate by nZVI occurs on the metal surface. And the total metal surface would strongly influence the kinetics of nitrate reduction. Thus, three different nZVI dosages $(0.2,2$ and $5 \mathrm{~g} / \mathrm{L})$ were employed to study the difference of kinetics of denitrification and the removal efficiency of nitrate (Fig. 4).

Fig. 5 showed clearly that the final removal efficiency of nitrate was higher when the nZVI dosage was higher. Nevertheless, when the dosage of nZVI was excess to $2 \mathrm{~g} / \mathrm{L}$, the final removal efficiency would increase slightly. The denitri-

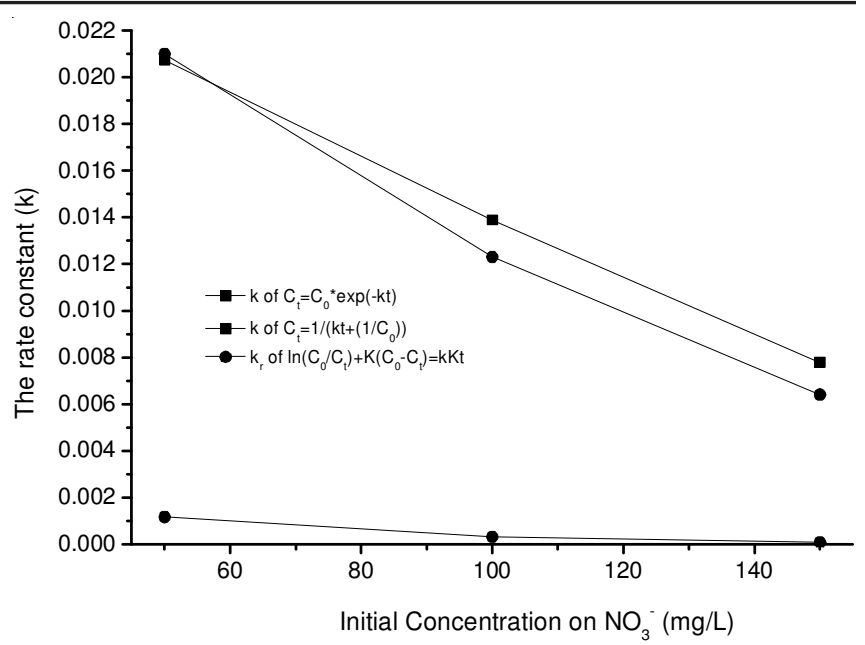

Fig. 3. Rate constants of the kinetic models estimation obtained at the at the initial nitration concentration of 50,100 and $150 \mathrm{mg} / \mathrm{L}$

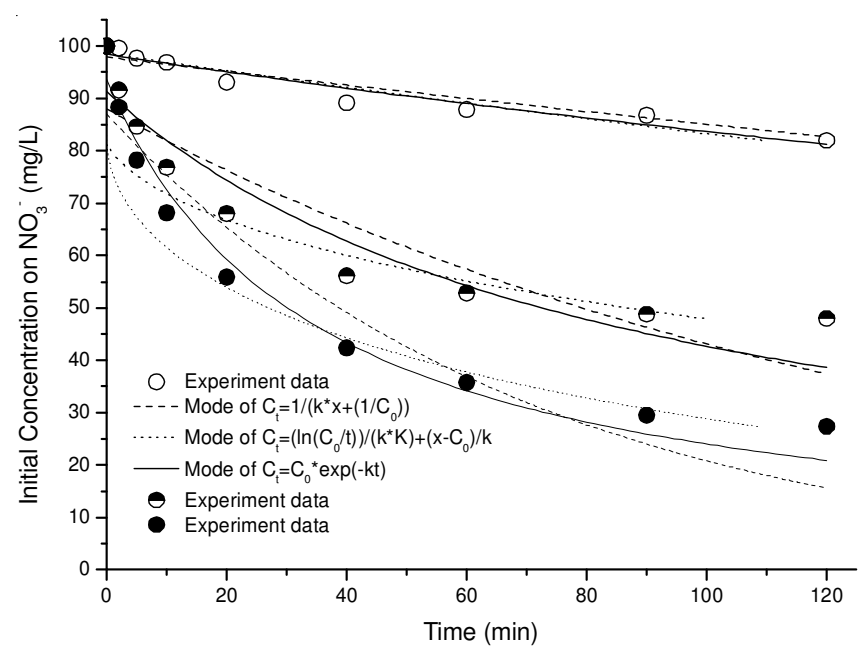

Fig. 4. Kinetic model estimation for nitration $(100 \mathrm{mg} / \mathrm{L})$ reduction at the $\mathrm{Fe} 0$ concentration of $0.2 \mathrm{~g} / \mathrm{L}$ (hollow circle), $2 \mathrm{~g} / \mathrm{L}$ (semi-solid circle) and $5 \mathrm{~g} / \mathrm{L}$ (solid circle). The dash lines stand for the model estimation of second-order kinetic. The dot lines stand for the model estimation of L-H kinetic. And the solid lines stand for the model estimation of first-order kinetic

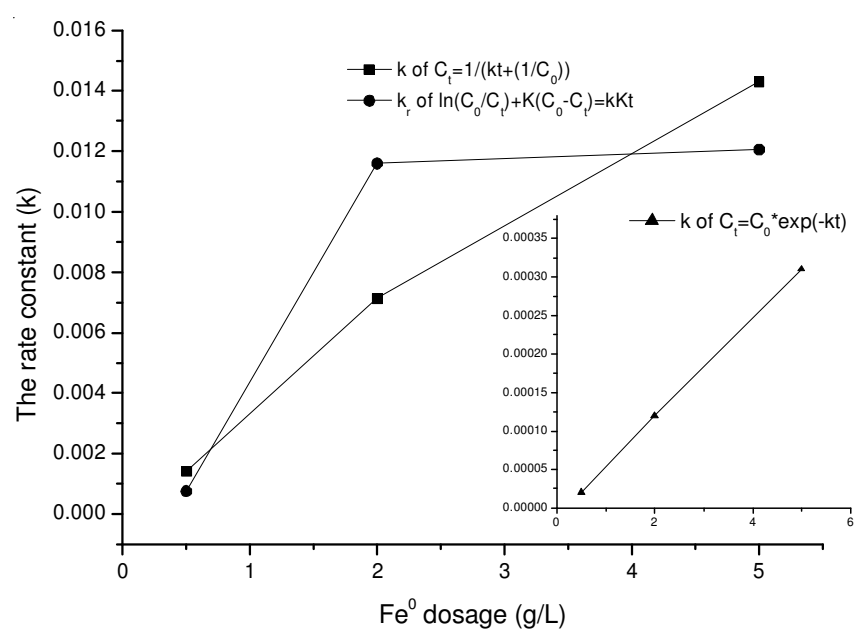

Fig. 5. Rate constants of the kinetic models estimation obtained at the nZVI concentration of $0.2,2.0$ and $5.0 \mathrm{~g} / \mathrm{L}$

fications at different nZVI dosage were also fit by F-, S- and L-H models (Fig. 4). The S-model could be used to fit the 
denitrification very well. The $\mathrm{R}^{2}$ values of $\mathrm{S}$ - model were higher than those of F- and L-H models (data were not shown). Moreover, when the dosage of nZVI was increased, the constant $\mathrm{k}$ of 3 models were also increase comply with linearity except for those of L-H models (Fig. 5).

Nitrogen mass balance during denitrification: The reaction between nitrate and $\mathrm{nZVI}$ is a true redox reaction. It is well known that nZVI would act as a reducing agent for nitrate. Its mechanisms are proposed by various researchers ${ }^{18,30}$. They have pointed out that the end-products could be nitrite, nitrogen gas, or ammonia depending on the experimental conditions.

In this study, it was founded that the concentration of ammonia increased continuously during the experiment as the decrease of nitrate. Nitrite concentration was increased obviously within started reaction time and then fluctuated (Fig. 6). The total $\mathrm{N}$ calculated as summation of remaining nitrate, nitrite and ammonia in the solution decreased during the whole reaction process. The loss of total $\mathrm{N}$ could reach $50 \%$ at the end of reaction $(2 \mathrm{~h})$. It implies that the part of the reaction products could be nitrogen gas, which was not analyzed for the open reaction system.

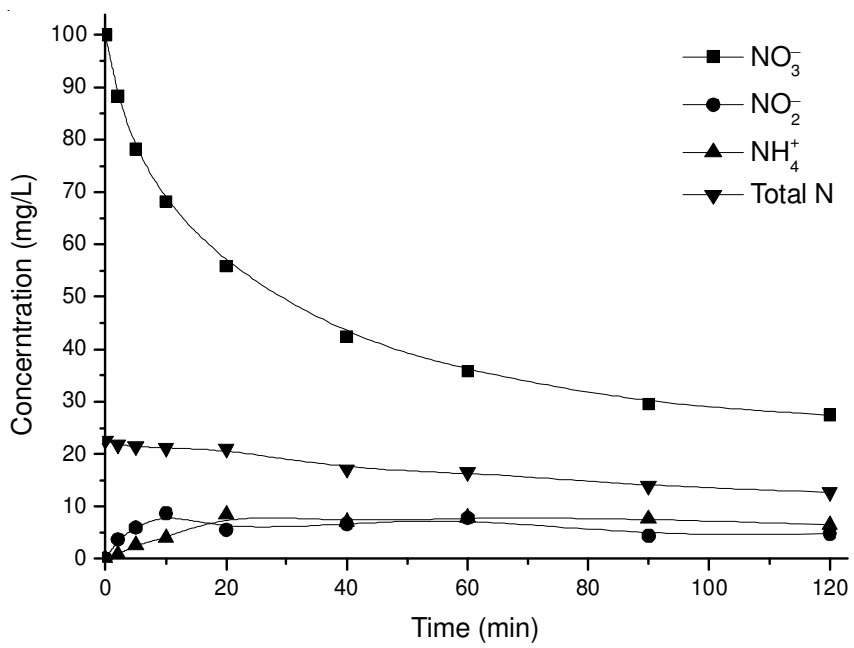

Fig. 6. Amount of various nitrogen species over hydraulic retention time during the nitrate reduction by nZVI

\section{Conclusion}

In this study, the preparation of nanoscale zero valent iron (nZVI) and reactivity of nZVI reaction in terms of nitrate reduction and the nitrogen mass balance during denitrification were evaluated. The nZVI particle was successfully produced in a co-solvent with water and ethanol, which were features of $44.8^{\circ}$ and $65.2^{\circ}$ diffraction peaks in the XRD pattern. The experimental data of nitrate reduction on the basis of nZVI were interpreted by F-, S- and L-H kinetic equations. The kinetic of nitrate removal by nZVI fitted well with the S- model in terms of $\mathrm{R} 2$ values and the constants of reaction. Ammonia and nitrogen gas were identified as the primary end-products from the reactions. Nitrite was produced rapidly during the started reaction of nitrate reducing and then reached equilibrium concentration.

\section{ACKNOWLEDGEMENTS}

The authors acknowledged the financial support by the National Natural Science Foundation of China (No. 21205001), the National 11th Five-Year Plan Major Scientific and Technological Issues of China (No. 2009ZX07316-004) and the Anhui Provincial Natural Science Foundation (1208085MB16).

\section{REFERENCES}

1. R.A. Crane and T.B. Scott, J. Hazard. Mater., 211-212, 112 (2012).

2. W.X. Zhang, J. Nanopart. Res., 5, 323 (2003).

3. A. Ghauch, A. Tuqan and H.A. Assi, Environ. Pollut., 157, 1626 (2009).

4. J. Fan, Y. Guo, J. Wang and M. Fan, J. Hazard. Mater., 166, 904 (2009).

5. S.H. Joo and D. Zhao, Chemosphere, 70, 418 (2008).

6. G. Naja, A. Halasz, S. Thiboutot, G. Ampleman and J. Hawari, Environ. Sci. Technol., 42, 4364 (2008).

7. X. Zhang, Y.M. Lin, X.Q. Shan and Z.L. Chen, Chem. Eng. J., 158, 66 (2010).

8. T. Cheng, Y.Z. Dai, C. Chen and Z.Q. Huang, Asian J. Chem., 24, 2579 (2012).

9. P. Varanasi, A. Fullana and S. Sidhu, Chemosphere, 66, 1031 (2007).

10. S. Choe, Y.Y. Chang, K.Y. Hwang and J. Khim, Chemosphere, 41, 1307 (2000).

11. Z. Xiong, D. Zhao and G. Pan, Water Res., 41, 3497 (2007).

12. Y. Xu and D. Zhao, Water Res., 41, 2101 (2007).

13. D. Karabelli, C. Uzum, T. Shahwan, A.E. Eroglu, I. Lieberwirth, T.B. Scott and K.R. Hallam, Ind. Eng. Chem. Res., 47, 4758 (2008).

14. S. Klimkova, M. Cernik, L. Lacinova, J. Filip, D. Jancik and R. Zboril, Chemosphere, 82, 1178 (2011).

15. S.R. Kanel, B. Manning, L. Charlet and H. Choi, Environ. Sci. Technol., 39, 1291 (2005).

16. W. Wang, Z.H. Jin, T.L. Li, H. Zhang and S. Gao, Chemosphere, 65 , 1396 (2006).

17. K.S. Lin, N.B. Chang and T.D. Chuang, Sci. Technol. Adv. Mater., 9, 1 (2008).

18. G.C. Yang and H.L. Lee, Water Res., 39, 884 (2005).

19. J.H. Zhanga, Z.W. Hao, Z. Zhang, Y.P. Yang and X.H. Xu, Process Saf. Environ., 88, 439 (2010).

20. J.M. Rodríguez-Maroto, F. García-Herruzo, A. García-Rubio, C. Gómez-Lahoz and C. Vereda-Alonso, Chemosphere, 74, 804 (2009).

21. Y.H. Hwang, D.G. Kim and H.S. Shin, J. Hazard. Mater., 185, 1513 (2011).

22. A. Ryua, S.W. Jeong, A. Jang and H. Choi, Appl. Catal. B-Environ., 105, 128 (2001).

23. Y.M. Chen, C.W. Li and S.S. Chen, Chemosphere, 59, 753 (2005).

24. S.C. Ahn, S.Y. Oh and D.K. Cha, J. Hazard. Mater., 156, 17 (2008).

25. F. Cheng, R. Muftikian, Q. Fernando and N. Korte, Chemosphere, 35, 2689 (1997).

26. C.P. Huang, H.W. Wang and P.C. Chiu, Water Res., 32, 2257 (1998).

27. Y.H. Huang and T.C. Zhang, Water Res., 38, 2631 (2004).

28. S. Choe, H.M. Liljestrand and J. Kim, Appl. Geo. Chem., 19, 335 (2004).

29. J. Andrieuxa, U.B. Demircia and P. Miele, Catal Today, 170, 13 (2011).

30. S.S. Chen, H.D. Hsu and C.W. Li, J. Nanoparticle Res., 6, 639 (2004). 